\title{
A cassava common mosaic virus vector for virus-induced gene silencing in cassava
}

\author{
Decai Tuo ${ }^{1,2}$, Peng Zhou ${ }^{1,2}$, Pu Yan ${ }^{1,2}$, Hongguang Cui ${ }^{3}$, Yang Liu ${ }^{1,4}$, He Wang ${ }^{1,4}$, Xiukun Yang ${ }^{1,4}$, Wenbin Liao ${ }^{1,2}$, \\ Di Sun ${ }^{1,4}$, Xiaoying $\mathrm{Li}^{1,2}$ and Wentao Shen ${ }^{1,2^{*}}$ (D)
}

\begin{abstract}
Background: Cassava is an important crop for food security and industry in the least-developed and developing countries. The completion of the cassava genome sequence and identification of large numbers of candidate genes by next-generation sequencing provide extensive resources for cassava molecular breeding and increase the need for rapid and efficient gene function analysis systems in cassava. Several plant virus-induced gene silencing (VIGS) systems have been developed as reverse genetic tools for rapid gene function analysis in cassava. However, these VIGS vectors could cause severe viral symptoms or inefficient gene silencing.

Results: In this study, we constructed agroinfection-compatible infectious cDNA clones of cassava common mosaic virus isolate CM (CsCMV-CM, genus Potexvirus, family Alphaflexiviridae) that causes systemic infection with mild symptoms in cassava. CsCMV-CM was then modified to a viral vector carrying the Nimble cloning frame, which facilitates the rapid and high-throughput cloning of silencing fragments into the viral genome. The CsCMV-based vector successfully silenced phytoene desaturase (PDS) and magnesium chelatase subunit I (ChII) in different cassava varieties and Nicotiana benthamiana. The silencing of the Chll gene could persist for more than two months.
\end{abstract}

Conclusions: This CsCMV-based VIGS system provides a new tool for rapid and efficient gene function studies in cassava.

Keywords: Cassava, Cassava common mosaic virus, Virus vector, Virus-induced gene silencing

\section{Background}

Cassava (Manihot esculenta Crantz, Euphorbiaceae) is native to the Amazon basin in South America, and its edible starchy storage root provides a major food source for nearly a billion people in tropical and subtropical regions [1, 2]. Recent data from the Consultative Group for International Agricultural Research (CGIAR) showed that cassava is becoming the second most important food crop in the least-developed countries and the fourth most

\footnotetext{
*Correspondence: shenwentao@itbb.org.cn

${ }^{1}$ Key Laboratory of Biology and Genetic Resources of Tropical Crops, Ministry of Agriculture and Rural Affairs \& Institute of Tropical Bioscience and Biotechnology, Chinese Academy of Tropical Agricultural Sciences, Haikou 571101, China

Full list of author information is available at the end of the article
}

important in developing countries, with a total production of 218 MT. Over half of the total production is in Africa and another third in Asia (https://www.rtb.cgiar. org/crops/cassava/). With increasing yields and technological innovations, cassava is not only an important food security crop but also an industrial and biofuel crop for production of industrial starch and ethanol in some countries [2].

Recent advances in next-generation sequencing technology have contributed to the completion of wholegenome sequences for wild and cultivated cassava lines $[3,4]$. Furthermore, large numbers of transcriptomic profiles have resulted in the identification of many candidate genes associated with cassava tissue development, metabolism, and responses to biotic and abiotic stress [5-8]. The accumulation of these genomic resources

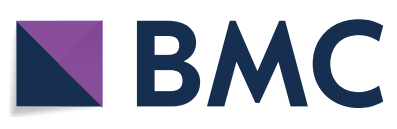

(c) The Author(s) 2021. This article is licensed under a Creative Commons Attribution 4.0 International License, which permits use, sharing, adaptation, distribution and reproduction in any medium or format, as long as you give appropriate credit to the original author(s) and the source, provide a link to the Creative Commons licence, and indicate if changes were made. The images or other third party material in this article are included in the article's Creative Commons licence, unless indicated otherwise in a credit line to the material. If material is not included in the article's Creative Commons licence and your intended use is not permitted by statutory regulation or exceeds the permitted use, you will need to obtain permission directly from the copyright holder. To view a copy of this licence, visit http://creativeco mmons.org/licenses/by/4.0/. The Creative Commons Public Domain Dedication waiver (http://creativecommons.org/publicdomain/ zero/1.0/) applies to the data made available in this article, unless otherwise stated in a credit line to the data. 
has increased the need for the development of reverse genetic technologies to identify functional genes that control desirable critical traits. The cassava genetic transformation technology is mature $[9,10]$, and available reverse genetic tools, such as RNA interference and geneediting, have been used to validate gene function by the stable genetic transformation [11-14]. However, cassava transformation is a laborious and lengthy process, and the protocols are not applicable to all cassava genotypes [10]. Virus-induced gene silencing (VIGS) as a powerful reverse genetic approach is a convenient and efficient alternative to genetic transformation [15]. In recent decades, more than 50 different plant DNA and RNA viruses and their viral satellites have been developed into VIGS vectors [16]. Through VIGS, many gene functions have been elucidated, including those involved in organ development, secondary metabolism, and responses to plant biotic and abiotic stresses [16]. In cassava, two mosaic geminiviruses, African cassava mosaic virus (ACMV) and East African cassava mosaic virus (EACMV), were developed into VIGS systems that have been reported to work efficiently in cassava [17-20]. However, ACMV and EACMV can cause characteristic chlorosis and distortion in cassava leaves $[15,19]$. These symptoms could interfere with the evaluation of VIGS effects and phenotypes in plants. Furthermore, viral vectors should observe strict importation and biosafety regulations [21]. The use of ACMV- and EACMV-based VIGS vectors is not permitted in certain countries, like China, because these viruses are not native to these areas and could easily cause pandemics. More recently, tobacco rattle virus (TRV, genus Tobravirus, family Virgaviridae)-based VIGS vector, which is widely used in Solanaceae, was used to silence the visible marker gene phytoene desaturase (PDS) in cassava, but the resulting photobleached phenotype was very weak $[22,23]$. Therefore, it is necessary to exploit new and more appropriate VIGS vectors for gene function analysis in cassava.

Cassava common mosaic virus (CsCMV) belongs to the genus Potexvirus (family Alphaflexiviridae). CsCMV was first reported in southern Brazil and is widespread in Latin America; it has also recently been found in China [24-26]. Compared with severe chlorosis and leaf distortion caused by cassava mosaic geminiviruses, the typical symptoms of CsCMV infections are generally milder mosaics with dark and light patches in cassava leaves [24, 27]. Thus, CsCMV is a candidate for use as a VIGS vector in cassava. Like other potexviruses, CsCMV contains a monopartite positive sense, single-stranded RNA genome 6,395 nucleotides (nt) in length excluding the $3^{\prime}$-poly-A tail [25]. Its genome comprises five open reading frames (ORFs) encoding an RNA-dependent RNA polymerase (RdRp), three triple gene block (TGB) proteins, and a coat protein $(\mathrm{CP})$. Several potexviruses, such as potato virus $\mathrm{X}$ (PVX), foxtail mosaic virus (FoMV), and pepino mosaic virus (PepMV), have been developed as vectors for expression of heterologous proteins [28-31] and transient loss-of-function studies based on VIGS in diverse dicot and monocot plant species [32-34].

We developed agroinfection-compatible infectious cDNA clones of CsCMV isolate CM (CsCMV-CM), which causes mild systemic symptoms in cassava. CsCMV-CM was modified to a viral vector carrying the Nimble cloning (NC) frame [35] that facilitates rapid and high-throughput insertion of gene-silencing fragments into the viral genome. We successfully silenced $P D S$ and magnesium chelatase subunit I (ChlI) genes in six different cassava varieties and Nicotiana benthamiana using the CsCMV-based vector. This vector will provide a new tool for rapid and efficient gene function studies in cassava.

\section{Results}

\section{Construction of the CsCMV-based agroinfectious clone and vector}

The full-length genomic complementary DNA (cDNA) of CsCMV-CM was obtained by PCR and then cloned between the CaMV $35 \mathrm{~S}$ promoter (35S P) and the poly(A) signal of T-DNA binary vector pGreenII-35S [36] to generate pCsCMV-CM using Gibson assembly (Fig. 1a). To test the infectivity of pCsCMV-CM, cassava plants were infiltrated with agrobacterium-carrying pCsCMV-CM. The pCsCMV-CM was infectious and induced mild mosaic symptoms of dark and light green patches in systemically infected cassava leaves ('SC 10') (Fig. 1b). The CsCMV-CM genomic RNA was detected in symptomatic cassava plants agroinoculated with $\mathrm{pCsCMV-CM}$ but not in mock-inoculated plants by RT-PCR (Additional file 1: Fig. S1).

To create a pCsCMV-CM VIGS vector based on a strategy similar to that used to construct the PVX and FoMV VIGS vectors [32, 37], a duplicated 90-bp putative CsCMV-CM $C P$ subgenomic promoter (SGP1) was added upstream of the authentic $C P$ promoter (SGP2) to drive target gene expression in the context of viral RNA (Fig. 1a). The SGP1 included a 60- nt upstream and a 30-nt downstream of the $C P$ start codon. Moreover, a Nimble Cloning (NC) frame sequence (adapter $1-S f i \mathrm{I}-c c d B$ gene-Sfi I-adapter 2) was inserted between the SGP1 and SGP2 to facilitate the rapid cloning of desired gene fragments for silencing, and the resultant vector was designated pCsCMVNC (Fig. 1a). Agroinfiltration of cassava plants with pCsCMV-NC showed that the vector was infectious and all 18 infiltrated plants in three independent experiments developed similar mild mosaic symptoms to 


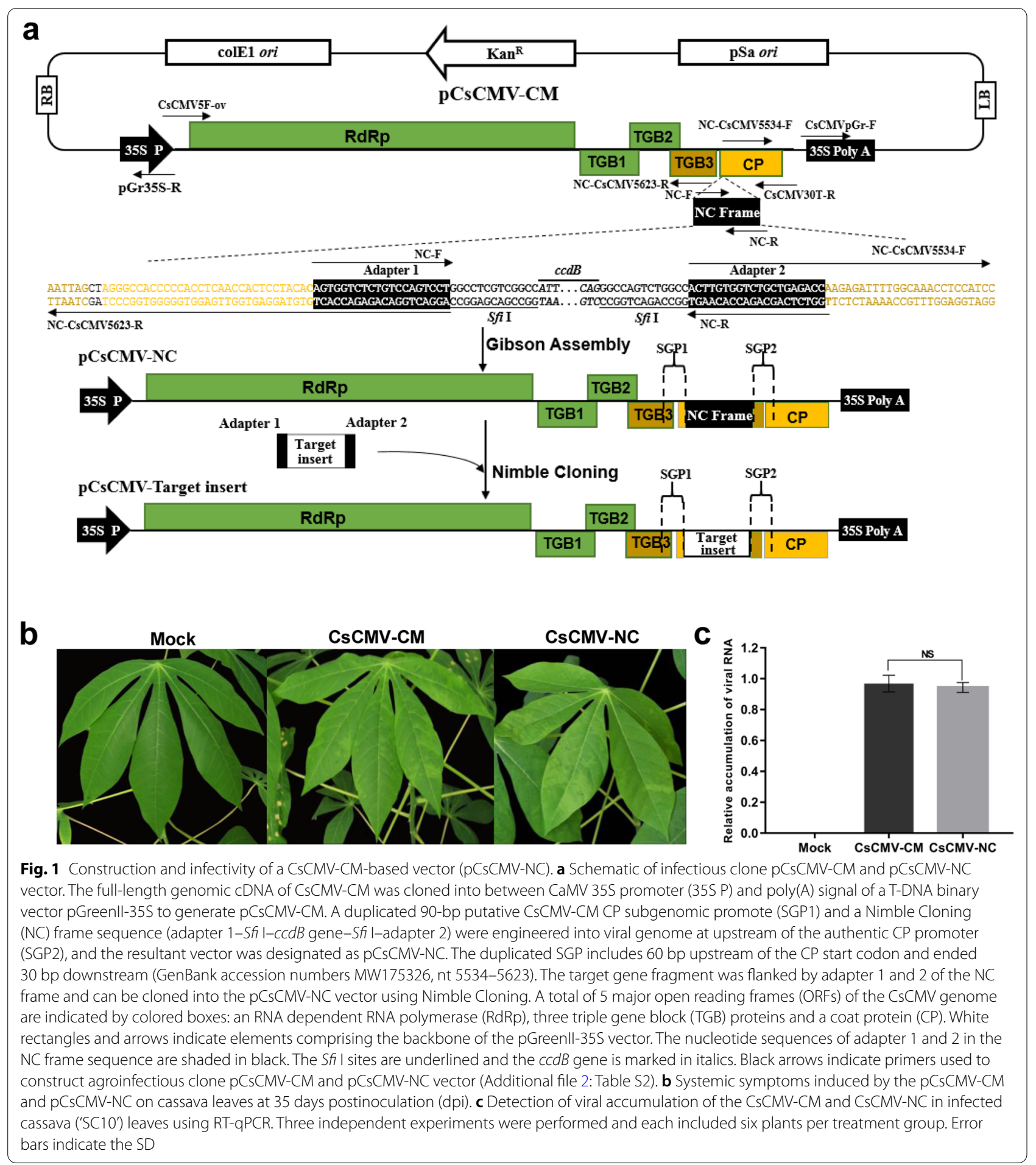

those of pCsCMV-CM-agroinfiltrated plants. The NC frame insertion was detected in systemic leaves by RTPCR (Additional file 1: Fig. S1). In addition, Reverse transcription quantitative real-time PCR (RT-qPCR) revealed that there was no significant difference in the accumulation of viral RNA in cassava plants infected with CsCMV-NC compared with that in plants infected with CsCMV-CM (Fig. 1c). Thus, insertion of SGP and the $\mathrm{NC}$ frame into $\mathrm{pCsCMV-CM}$ did not affect the viral infectivity. Accordingly, each PCR-generated target 
fragment with $\mathrm{NC}$ adapters could be cloned into the pCsCMV-NC vector via Nimble Cloning.

\section{Silencing of cassava PDS and Chll genes using the PCsCMV-NC vector}

To test whether pCsCMV-NC could be used to induce endogenous gene silencing in cassava, we first silenced two VIGS marker genes, PDS and Chll. After genomewide, off-target gene-silencing analysis, 487-bp PDS and 345-bp ChlI DNA fragments were cloned into PCsCMV$\mathrm{NC}$ in an antisense orientation to generate the pCsCMV$\mathrm{PDS}_{487}$ and pCsCMV-ChlI 345 vectors, respectively. The 3-week-old cassava plants agroinfiltrated with pCsCMV$\mathrm{PDS}_{487}$ or pCsCMV-ChlI 345 initially exhibited mild photobleaching or a yellow-leaf phenotype in the veins of the second and third leaves above the inoculated leaves at 15 $\mathrm{dpi}$, and developed severe photobleaching or a yellowing VIGS phenotype in the stems and the newly emerging leaves at 35 dpi (Fig. 2a). Similarly, the silencing phenotype occurred in the upper systemic leaves of 5-monthold cassava plants agroinoculated with pCsCMV-PDS or pCsCMV-ChlI ${ }_{345}$ (Fig. 2b). According to the number of leaves and areas exhibiting silencing phenotypes, silencing was more effective in the CsCMV-ChlI $\mathrm{C}_{345^{-}}$ infected plants than in the CsCMV-PDS ${ }_{487}$-infected plants. The CsCMV-ChlI 345 -induced silencing phenotype could be maintained more than two months, whereas the photo-bleached leaves infected with CsCMV-PDS 487 tended to senesce at $45 \mathrm{dpi}$ (Additional file 1: Fig. S2). RT-qPCR showed that the PDS and ChlI mRNA levels were reduced by approximately $70 \%$ and $81 \%$ in the silenced leaves compared with those in the CsCMV-NCinfected leaves, respectively (Fig. 2c). These results suggest that the pCsCMV-NC vector can be used to silence endogenous genes in cassava.

\section{Silencing effects of different sizes of Chll genes inserted

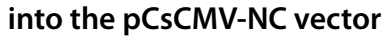

To investigate whether the insert size affects VIGS efficiency, additional CsCMV-based VIGS constructs of different sizes carrying partial ChlI genes $(133,236$, and $439 \mathrm{bp}$ ) were created. The resulting pCsCMV-ChII ${ }_{133}$, - ChlI $_{236}$, and $-\mathrm{ChlI}_{439}$ and $\mathrm{CsCMV}-\mathrm{ChlI}_{345}$ constructs were used to separately agroinoculate 5-month-old cassava plants. During the following 30-d observation period, plants infected with CsCMV-ChlI ${ }_{345}$ or CsCMV$\mathrm{ChlI}_{439}$ showed similar strong silencing phenotypes with larger areas of yellowing in most new leaves; a milder and unambiguous yellow-colored VIGS phenotype was observed in plants infected with CsCMV-ChlI 236 and ChlI $_{133}$, respectively (Fig. 3a). RT-qPCR analysis confirmed that relative expression levels of ChlI mRNA were reduced by $53.7 \%, 60 \%, 81.7 \%$ and $83 \%$ in the leaves exhibiting the yellow phenotype induced by CsCMV$\mathrm{ChlI}_{133},-\mathrm{ChlI}_{236}$, and $-\mathrm{ChlI}_{439}$ and CsCMV-ChlI 345 , respectively, compared with those in the CsCMV-NCinfected leaves (Fig. 3c). These results suggest that the insert size of target gene was related to the silencing effects.

\section{Stability of the 345-bp Chll fragment in pCsCMV-Chll ${ }_{345}$} At $70 \mathrm{dpi}$, we observed the different VIGS silencing effects in 12 leaves from the agroinfiltrated leaf to the top-most leaf (L1 to L12, numbered from the agroinfiltrated to the upper leaves) in the CsCMV-ChlI ${ }_{345^{-}}$ infected cassava plants. The obvious silencing phenotype was maintained in the third (L3) to eighth (L8) leaves, and the phenotype gradually became less severe in the upper leaves, almost disappearing in the top leaves (Fig. 3b). To investigate the stability of the Chll fragment in pCsCMV-ChlI ${ }_{345}$ in the leaves of different ages, RTPCR was carried out. It revealed that the ChlI insertions were relatively stable in L1 (agroinfiltrated leaf) because the expected 792-bp band was specifically amplified. However, an additional smaller band of $315 \mathrm{bp}$, whose size was similar to that of the empty pCsCMV-CM vector appeared in leaves L2 to L10 and became more obvious in the upper systemically infected leaves (Fig. 3d); this suggested that the inserted $\mathrm{ChlI}_{345}$ was partially lost to different extents in these leaves. Leaves L11 to L12 without silencing phenotypes exhibited complete deletions of the ChlI fragment because only the 315-bp band was detected (Fig. 3d).

\section{The CsCMV VIGS vector is suitable for different cassava cultivars}

To test whether our CsCMV-NC VIGS vector could induce silencing in other cassava varieties, five additional

\footnotetext{
(See figure on next page.)

Fig. 2 Silencing of PDS and Chll genes in cassava using the CsCMV-based vector. $\mathbf{a}$, Representative silencing phenotypes in 3-week-old cassava ('SC10') leaves and stems induced by silencing of PDS or Chll using the CsCMV-based VIGS vector carrying a fragment of cassava PDS (pCsCMV-PDS ${ }_{487}$ ), ChIl (pCsCMV-Chll ${ }_{345}$ ) or control vector (pCsCMV-NC) at 15 and 35 dpi. b Silencing phenotypes in 5-month-old cassava plants ('SC10') induced by infection with pCsCMV-PDS 487 or pCsCMV-Chll ${ }_{345}$ at 35 dpi. c RT-qPCR analyses of PDS and Chll mRNA expression in 3-week and 5-month-old cassava plants infected with CsCMV-PDS ${ }_{487}, \mathrm{CsCMV}_{\mathrm{C}} \mathrm{Ch} \mathrm{Il}_{345}$ and CsCMV-NC. Statistical tests were performed using Student's $t$ test, compared with plants infected with non-target control CsCMV-NC ( ${ }^{* * P}<0.01$ and $\left.{ }^{* *} P<0.001\right)$. Three independent experiments were performed, and each included six plants per treatment group. Error bars indicate the SD
} 

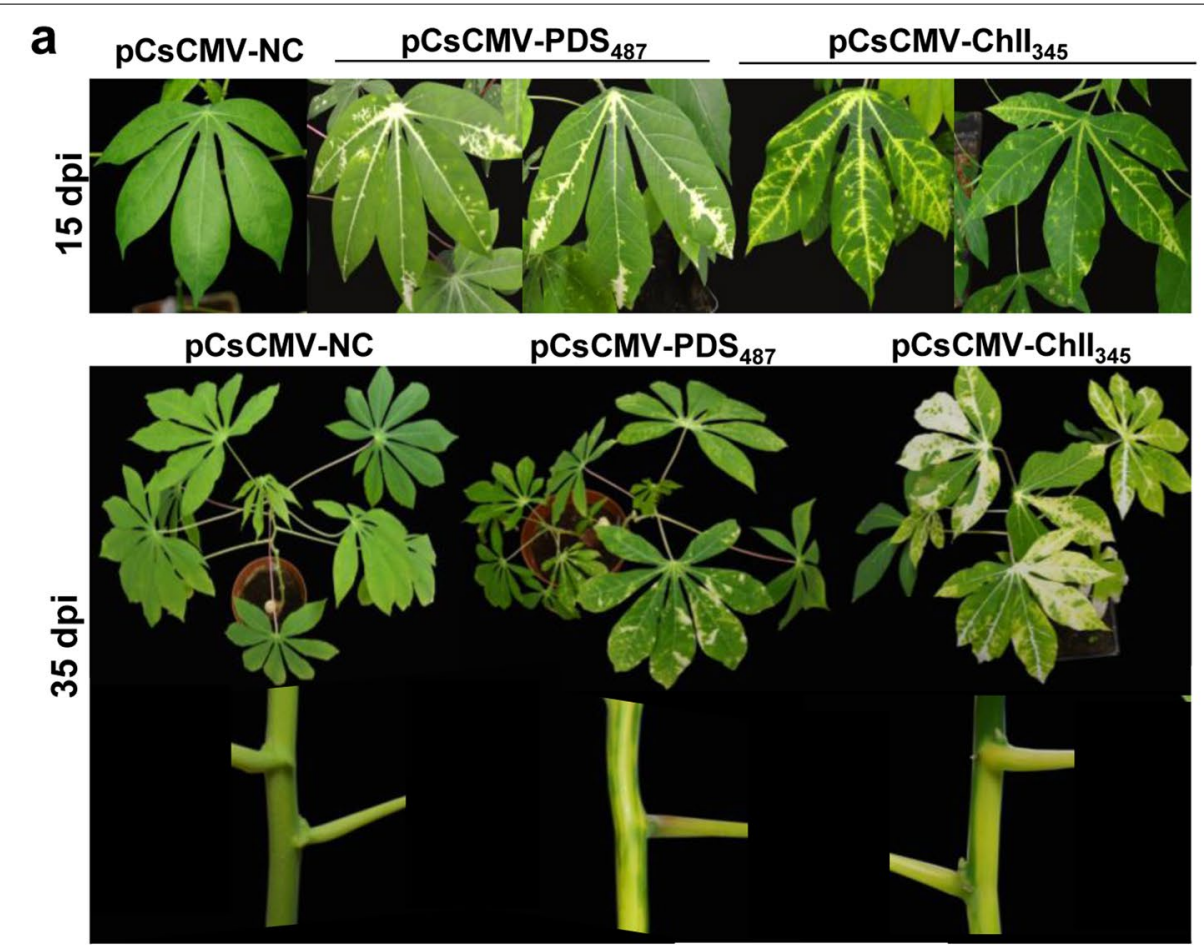

b

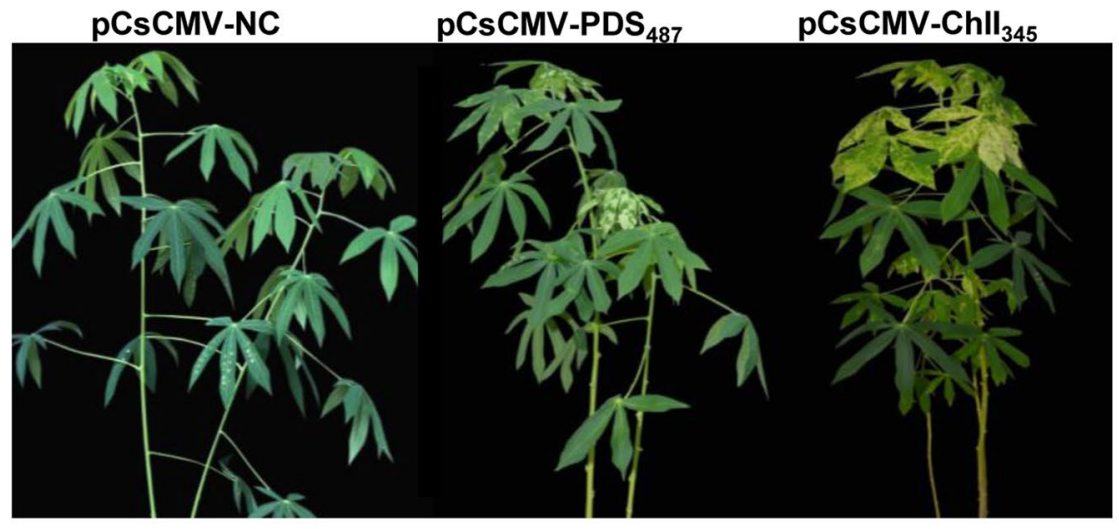

C

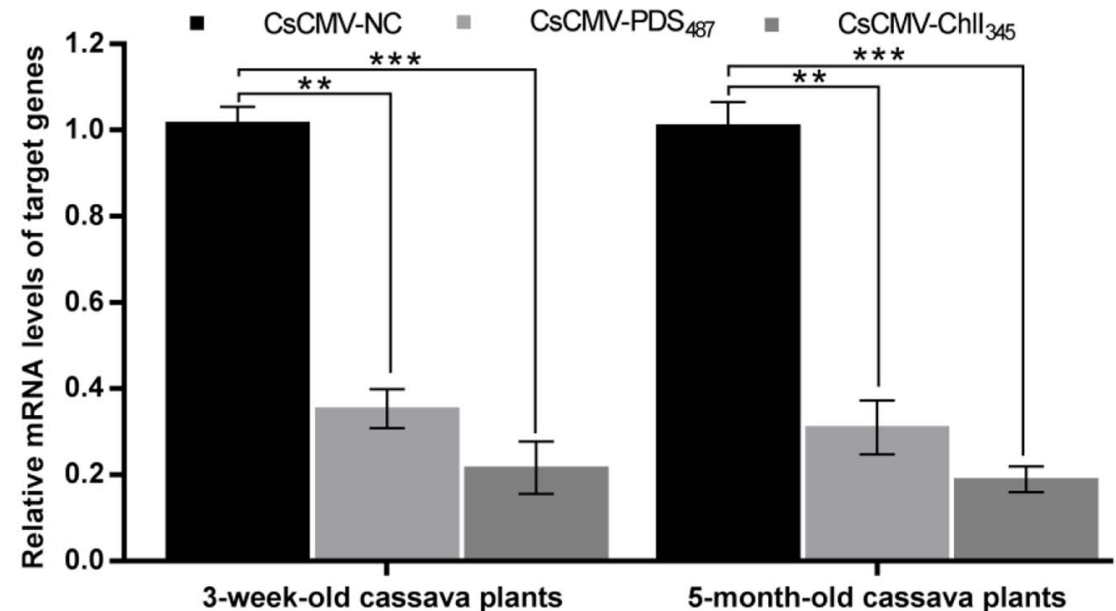

Fig. 2 (See legend on previous page.) 


\section{a}

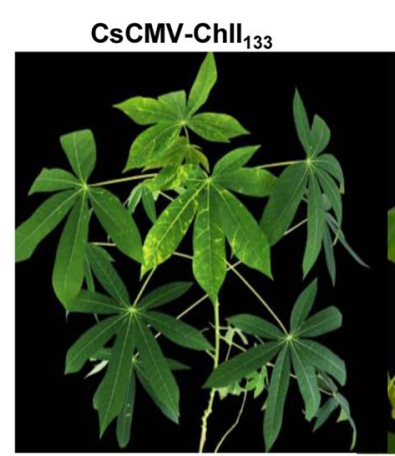

b
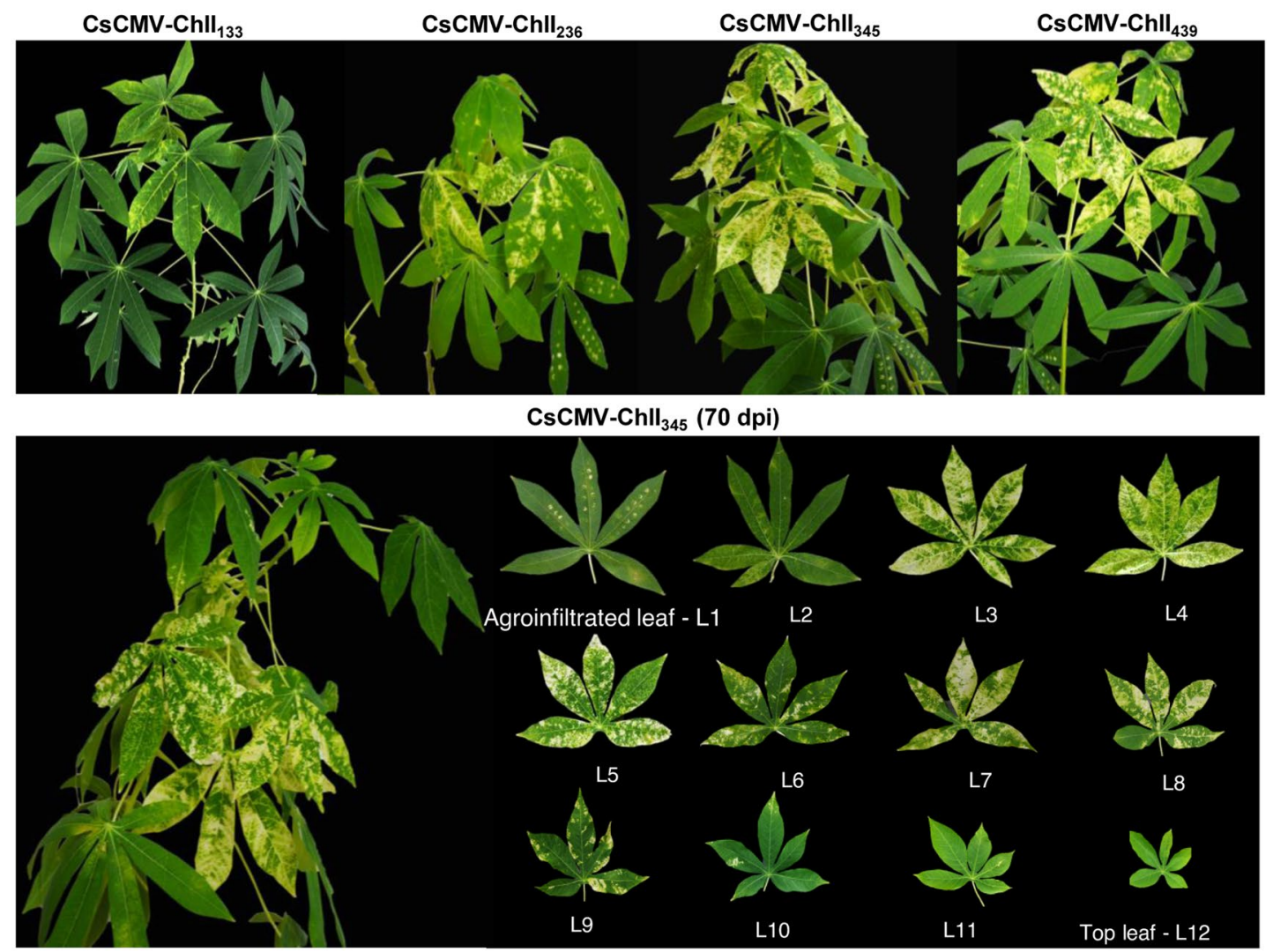

CsCMV-ChII ${ }_{345}$ (70 dpi)
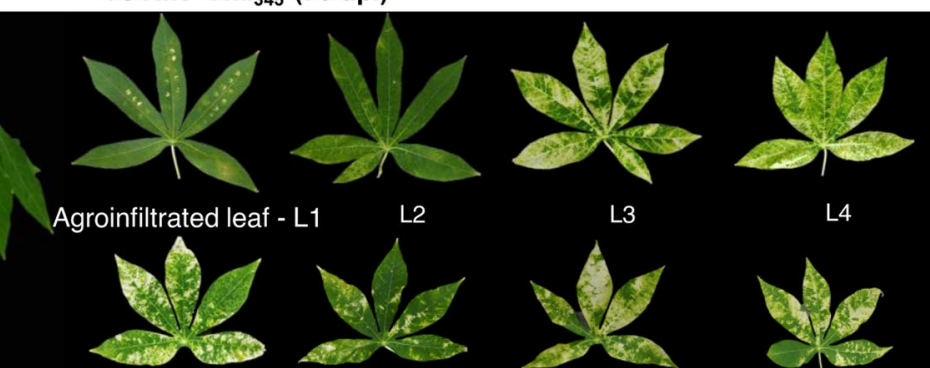

L5

L6

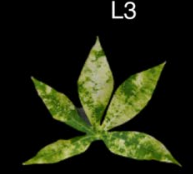

L4

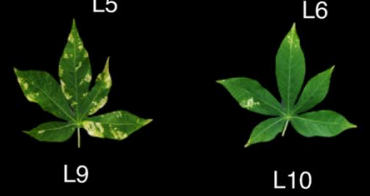

L7

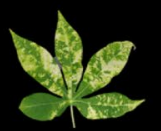

L8
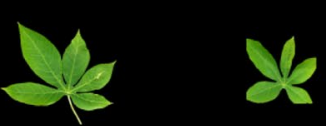

L11

Top leaf $-L 12$

C

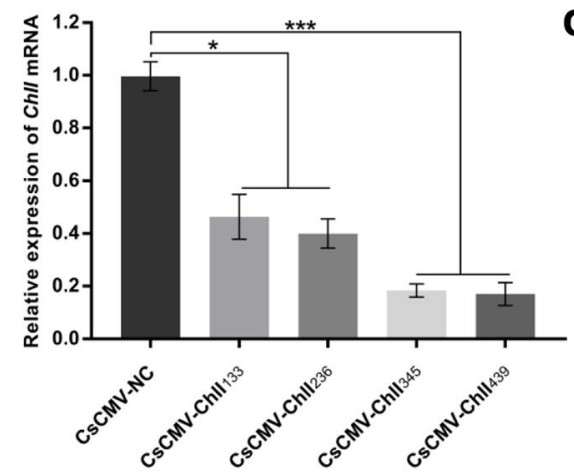

d

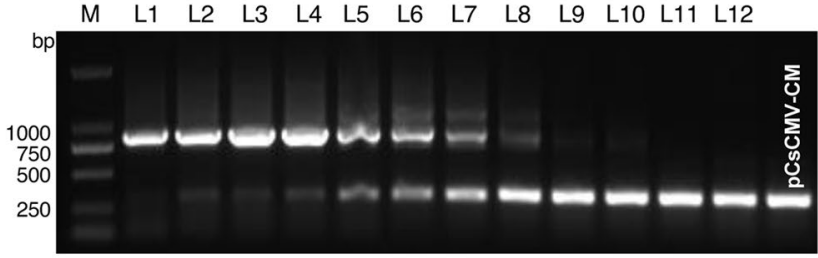

Fig. 3 Silencing effects and stability of insert size of Chll gene in the CsCMV-based VIGS vectors. a 5-month-old cassava plants ('SC10') were infected with the CsCMV -based VIGS vectors carrying different sizes (133, 236, 345 and 439 bp) of partial Chll and representative silencing phenotypes by silencing of Ch/l were photographed at $35 \mathrm{dpi}$. b Silencing phenotypes in the different-aged leaves induced by silencing of Ch/l using the pCsCMV-Chll 345 at 70 dpi. c RT-qPCR analyses of Ch/l mRNA expression in 5-month-old cassava plants infected with the CsCMV-based VIGS vectors carrying different sizes $(133,236,345$ and 439 bp) of partial Chll. Satistical tests were performed using Student's $t$ test, compared with the plants infected with non-target control CsCMV-NC ( ${ }^{*} P<0.05$ and $\left.{ }^{* * *} P<0.001\right)$. Three independent experiments were performed and each included six plants per treatment group. Error bars indicate the SD. $\mathbf{d}$ RT-PCR analyses of the stability of the 345-bp Ch/l fragment in pCsCMV-Chll 345 among the different-aged leaves. A total of 12 leaves from the agroinfiltrated leaf to the top-most leaf ( $L 1$ to $L 12$, numbered from the inoculated leaves) were collected for RNA extraction and RT-PCR analysis. The non-target control pCsCMV-NC was used as the control

lines popular in China $(60,444, \mathrm{ZM} 9781, \mathrm{SC} 5, \mathrm{SC} 8$, and SC9) were agroinfiltrated with the pCsCMV-NC and pCsCMV-ChlI $I_{345}$ vectors. All five of these lines were susceptible to pCsCMV-NC, exhibiting mild mosaic symptoms, and pCsCMV-ChlI 345 induced the typical yellow-white phenotype to different extents in new leaves of all lines at 30 dpi (Fig. 4a). RT-qPCR showed that the mRNA levels of ChlI were decreased by approximately 

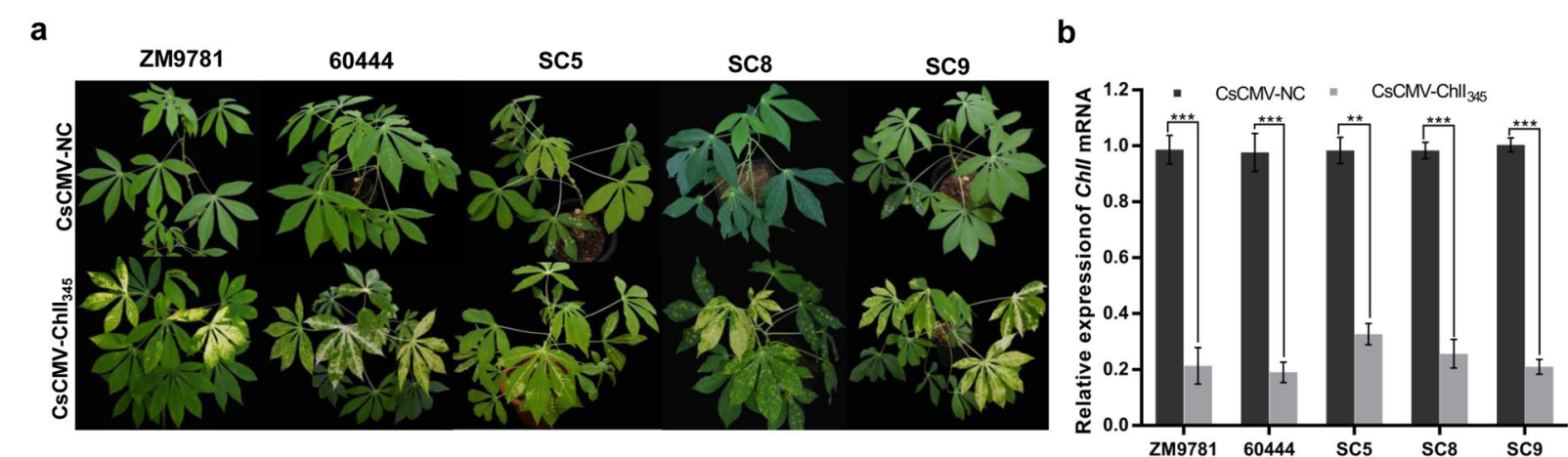

Fig. 4 Infection and VIGS phenotypes in 5 cassava cultivars agroinfiltrated with pCsCMV-Chll 345 and pCsCMV-NC. a Silencing phenotypes in 5 cassava cultivars $\left(60,444\right.$, ZM9781, SC5, SC8, and SC9) using the pCsCMV-NC and pCsCMV-Chl ${ }_{345}$ at 30 dpi. b RT-qPCR analyses of Ch/l mRNA expression in 5 cassava cultivars infected with pCsCMV-Chll ${ }_{345}$ and pCsCMV-NC. Satistical tests were performed using Student's $t$ test, compared with plants infected with non-target control pCsCMV-NC ${ }^{* *} P<0.01$ and $\left.{ }^{* * *} P<0.001\right)$. Three independent experiments were performed and each included six plants per treatment group. Error bars indicate the SD

68 to $81 \%$ in these five cultivars infected with CsCMV$\mathrm{ChlI}_{345}$ compared with those in the CsCMV-NC-infected control plants (Fig. 4b).

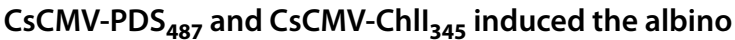 and chlorotic VIGS phenotypes in N. benthamiana}

Like cassava, the model plant $N$. benthamiana can be systemically infected with CsCMV [25]. The 487-bp fragment of cassava PDS and 345-bp fragment of cassava ChlI in pCsCMV-PDS 487 and pCsCMV-ChlI 345 shared $75.7 \%$ and $82.6 \%$ similarity with $N$. benthamiana PDS and ChlI, respectively (Additional file 1: Fig. S3). Agroinfiltration of $N$. benthamiana with
pCsCMV-PDS $_{487}$ and pCsCMV-ChlI ${ }_{345}$ resulted in the appearance of photo-bleached spots and chlorosis, respectively (Fig. 5a). These silencing phenotypes could be maintained longer than two months after inoculation. RT-qPCR results showed that PDS and ChlI mRNA expression in the $N$. benthamiana plants infected with CsCMV-PDS 487 and CsCMV-ChlI ${ }_{345}$ were only $26.7 \%$ and $17 \%$ of those in the CsCMV-NCinfected plants, respectively (Fig. 5b). These results suggest that the PDS and ChlI fragments from cassava could induce silencing of the corresponding orthologous genes in $N$. benthamiana via the CsCMV-based VIGS vector. a

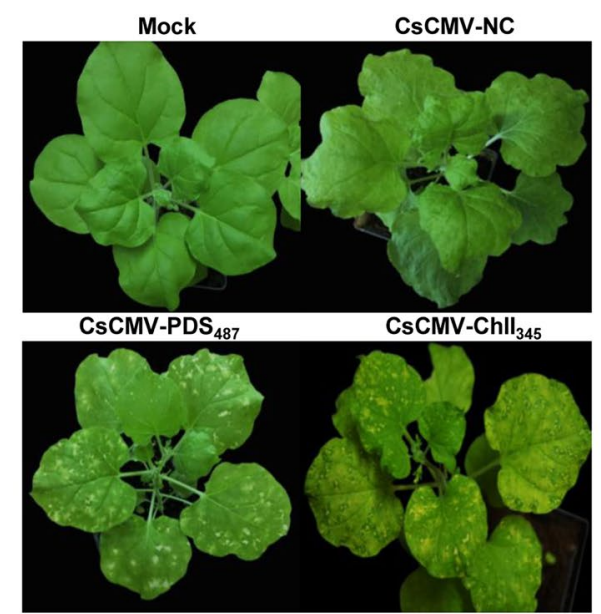

b

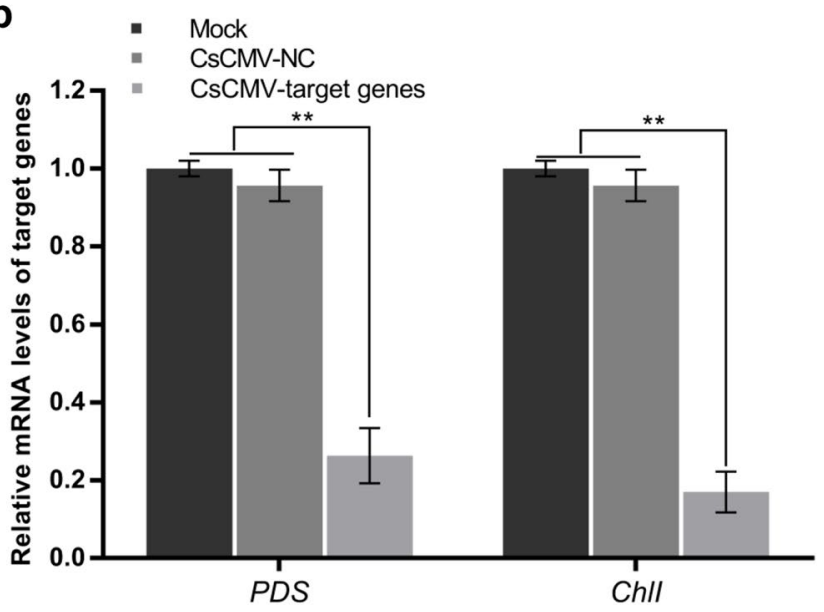

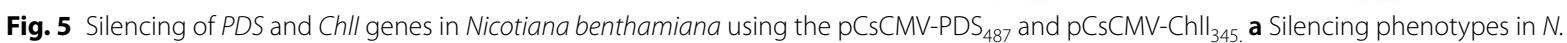
benthamiana using the pCsCMV-PDS 487 and pCsCMV-ChIl 345 at 25 dpi. b RT-qPCR analyses of PDS and Ch/l mRNA expression in N. benthamiana infected with pCsCMV-PDS 487, pCsCMV-ChII 345 and pCsCMV-NC. Satistical tests were performed using Student's $t$ test, compared with plants infected with non-target control pCsCMV-NC (**P<0.01). Three independent experiments were performed and each included six plants per treatment group. Error bars indicate the SD 


\section{Discussion}

In this study, we developed a VIGS vector derived from CsCMV-CM for use in cassava plants. The CsCMV-based vector has several advantages over previous ACMV, EACMV, and TRV VIGS vectors in cassava [17-19, 23]. First, the vector caused milder mosaic symptoms than ACMV and EACMV in cassava leaves, making it suitable for functional genomics in cassava because severe viral symptoms may be confused with the effects of the VIGS vector and its resulting phenotypes in test plants. Second, unlike ACMV and EACMV and TRV, which consist of bipartite DNA and RNA genomes, respectively, CsCMV has a single-stranded RNA genome. A virus vector-based single viral genome is usually easier to manipulate than those of multipartite genomes [33]. For example, compared with vectors based on multipartite viruses, agroinfiltration with CsCMV vectors does not require the preparation of a mixture of agrobacterium suspensions, each one carrying discrete portions of the genome. Third, CsCMV spreads by mechanical transmission, which makes infection of plants easier through leaf agroinfiltration, resulting in effective VIGS. Conversely, agroinfiltration of cassava plants with geminivirus-based vectors requires injection of agrobacteria suspensions near the axillary buds and through superficial cuts in the stem. This inoculation method could damage the meristems and affect plant growth [19]. Biolistic delivery has also been used for geminivirus inoculation of cassava plants, but it is a high-cost method [18]. In this study, $100 \%$ infection efficiency by agroinfiltration of pCsCMV$\mathrm{NC}$ vector was achieved. Fourth, ACMV and EACMV are transmitted by whitefly. Their viral vectors have a potentially higher risk of escape into the environment than the CsCMV vector by mechanical transmission. Fifth, we inserted the $\mathrm{NC}$ frame of Nimble Cloning into the CsCMV genome to facilitate the rapid cloning of desired target genes. Accordingly, each PCR-generated target fragment with $\mathrm{NC}$ adapters can be cloned into a circular pCsCMV-NC vector via a simple mixture of the rarecutting restriction enzyme $S f i$ I and T5 exonucleases, which simultaneously accomplish linearization of the vector and the ligation reaction [35]. In previous studies, Gateway-based and ligation-independent VIGS vectors have been used for rapidly cloning a target fragment without multiple digestion and ligation steps [22, 38]. In contrast to these approaches, Nimble Cloning does not require an additional step to linearize the VIGS vector; therefore, it is simpler and more cost-effective than Gateway-based and ligation-independent cloning methods [35]. Furthermore, the NC frame includes the $c c d B$ gene, which is a positive selection marker to facilitate more rapid and accurate screening of putative recombinant colonies. A similar strategy is widely applied in Gateway methods [22, 38]. In this study, greater than $95 \%$ of clones were positive in each transformation using Nimble Cloning with mixtures of pCsCMV-NC vector and PCR amplicons of individual target gene fragments. Thus, the CsCMV-based VIGS system could also be applied in the construction of a cassava VIGS library for high-throughput forward genetics screening in the future.

Application of CsCMV as a VIGS vector requires the insertion of foreign sequences into the viral genome at positions that do not affect viral infectivity. CsCMV is the type member of the genus Potexvirus. There are two strategies for construction of potexvirus-based vectors according to their viral genome organization. Introduction of an additional SGP upstream of the CP gene for expression of a gene of interest is a commonly used approach and has successfully been used in PVX, PepMV, and FoMV [31-33]. The other strategy is development of a FoMV-based VIGS vector by insertion of the cloning site after the CP stop codon [34]. In our study, we constructed the CsCMV vector by duplicating the 90-bp putative CsCMV CP SGP, including the potexvirusspecific octanucleotide motif (GUUAAGUU) [37]. Our result showed that engineering the duplicated copy of the putative CsCMV CP SGP and NC cloning frame into the viral genome did not affect infectivity of the CsCMV$\mathrm{NC}$ vector in cassava and $N$. benthamiana. Moreover, the anti-sense fragments of PDS and ChlI cloned into the pCsCMV-NC vector caused obvious silencing phenotypes in both host plants.

In this study, the 487-bp fragment of $P D S$ and the 345-bp fragment of ChlI with best target region score were selected by genome-wide off-target gene silencing assessment [39]. The predicted result showed that ChlI $_{345}$-derived siRNAs can target two ChlI homologous genes located on the 16th and 17th chromosomes of cassava while the predicted siRNAs only can match $P D S$ gene in the 5th chromosome of cassava. More target sites might result in more accumulation of the target fragment-derived siRNAs and induced more severe silencing phenotype. Indeed, we observed that the $\mathrm{ChlI}_{345}$-silenced cassava plants exhibited more severe silencing phenotype with large areas of yellow-white leaf than $P D S_{487}$-silenced cassava plants. Certainly, the gene silencing efficiency is related to various factors including sequence space, target availability, the position of nucleotides, secondary structures of mRNA and intrinsic characteristics of siRNA and target mRNA [40]. In addition, we assessed whether the size of the host-derived sequence insert affects CsCMV based VIGS efficiency. Our results showed that the CsCMVvectors carrying partial ChlI genes of different sizes $(133,236,345$, and $439 \mathrm{bp})$ in antisense orientation could effectively induce silencing in cassava, and the 
more severe silencing phenotype was observed when the insert length was more than 300 bp. Similarly, infection with the PVX VIGS vector harboring PDS sequences of 412-bp in antisense orientation resulted in strong photobleaching phenotypes in both diploid and cultivated tetraploid Solanum species [32]. However, FoMV vector with a duplicated FoMV CP SGP was used to induce effective silencing of endogenous genes in barley when target sequence insert was a short inverted-repeat fragment but not an antisense one [33]. Therefore, the effect of length of target genes on silencing depends on the different potexviruses-derived vectors and hosts.

As VIGS approaches induce transient gene knockdowns, increasing the duration of endogenous gene silencing will widen the application of VIGS in functional genomics. In this work, strong yellow-white silencing phenotype in systemic leaves infected with CsCMV-ChlI ${ }_{345}$ can persist for more than two months. The longer silencing period will facilitate characterization of the gene functions involved in developmental and biosynthetic pathways and stress tolerance in cassava. However, the phenotype gradually became less severe in the upper leaves and almost disappeared in the top leaves, which was related to partial or complete loss of inserted $\mathrm{ChlI}_{345}$ fragment because of the sequence redundancy of the duplicated SGP in potexvirus-based vectors [34, 37]. To address the problem, an PVX-based expression vector was improved to stabilize the foreign inserts by replacing the duplicated SGP with a heterologous SGP combined with an N-terminal CP deletion [37]. In addition, change of the position of insertion was used to increase stability of the insert. The cloning site in FoMV was placed after the stop codon following the CP coding sequence instead a duplicated subgenomic promote [34]. However, the loss of $P D S$ inserts still occurred when this FoMV vector was used to silence PDS in maize [34]. Therefore, the stability of sequences inserted into viral genomes is regard as a surprisingly complex problem involved in the genome characteristics, the host environment and the demography of a virus population [41].

Over the years, numerous cassava varieties with different traits have been released in the world [2]. Theoretically, CsCMV-based VIGS system are applicable to cassava lines susceptible to CsCMV. Here, we induced ChlI gene silencing in 6 popular lines $(60,444$, ZM9781, SC5, SC8, SC9 and SC10) in China using pCsCMV$\mathrm{ChII}_{345}$, which will contribute to use this vector to analysis some functional genes involved in important biological and agronomical traits among these cultivars. In addition, CsCMV were detectable in fibrous and storage roots of CsCMV-NC-infected cassava plants (Additional file 1: Fig. S4), thereby we will further broaden the use of CsCMV vector in gene silencing from leaves to root tissues like ACMV-based vector.

\section{Conclusions}

We developed an effective CsCMV-based VIGS vector that induced endogenous gene silencing in different cassava cultivars. Target fragments for gene silencing can easily be cloned into the CsCMV vector using onestep Nimble cloning. The new VIGS system will facilitate rapid and high-throughput loss-of-function studies in cassava.

\section{Methods}

\section{Generation of a CsCMV agroinfectious clone}

Total RNA was extracted from the CsCMV-infected cassava leaves displaying mild mosaic symptoms in a germplasm garden in Chengmai (CM) of Hainan Province, China. The first-strand cDNA was synthesized from $1.0 \mu \mathrm{g}$ of total RNA with the Takara RNA PCR Kit (AMV) Ver. 3.0 (TaKaRa, Japan) using random 9 mers and oligo dT-Adaptor primers. The complete genome sequence of the CsCMV isolate, designated CsCMV-CM, were determined by RT-PCR and SMARTer $5^{\prime} / 3^{\prime}$ RACE kits (TaKaRa) based on our recent study [25], respectively. All primers used for PCR amplification of viral genome are listed in Additional file 2: Table S1. This whole genome sequence of CsCMV-CM has been deposited in GenBank under the accession number MW175326.

The full-length viral sequence and the backbone fragment of pGreenII-35S vector were individually PCRamplified using CsCMV-CM cDNAs and pGreenII-35S plasmid [36] as templates with two primer pairs CsCMV5Fov/CsCMV30T-R and CsCMVpGr-F/pGr35S-R which shared 25-36 homologous bases at each end (Additional file 2: Table S2). Then, both overlapping PCR products were mixed and assembled to generate $\mathrm{pCsCMV-CM}$ according to the instructions of Gibson Assembly Cloning Kit (NEB, USA). Briefly, 100 ng each purified PCR fragment and $5 \mu \mathrm{L} 2 \times$ Gibson mix (NEB) was incubated at $50{ }^{\circ} \mathrm{C}$ for $1 \mathrm{~h}$, and then placed on ice for Escherichia coli strain $\mathrm{DH} 5 \alpha$ transformation. The resultant clones were confirmed by PCR with primer pair CsCMV5259F/ CsCMV3R and DNA sequencing. Similarly, three overlapping DNA fragments (I, II, and NC) were amplified in separate PCRs to construct the pCsCMV-NC. The DNA fragment I containing the replicase, the $T G B$ genes and the duplicated 90-bp putative CsCMV-CM CP SGP1 was amplified from pCsCMV-CM using the primers CsCMV5Fov/NC-CsCMV5623-R. The SGP1 contained the 60-nt upstream and 30-nt downstream of the CP start codon. The pCsCMV-CM was used as the template with the primers NC-CsCMV5534-F/pGr35S-R to amply the DNA fragment II covering the authentic $\mathrm{CP}$ promoter 
SGP2 and the backbone fragment of pGreenII-35S. The $\mathrm{NC}$ frame from pNC-UC vectors [35] were amplified using primer pairs NCF/NCR. All primer pairs used Gibson Assembly included sequences overlapping adjacent fragments by 21 to $25 \mathrm{nt}$. The Gibson Assembly reaction of pCsCMV-NC is same as described for the construction of pCsCMV-CM. The transformation was performed using Escherichia coli strain DB3.1.

\section{Generation of $\mathrm{Cs} C M V$ VIGS constructs}

The regions of target genes for genome-wide off-target gene silencing were selected using SGN VIGS Tool [39]. A 487 bp cassava PDS fragment of (GenBank accession: XM_021757403) and four partial cassava Chll fragments (GenBank accession: XM_021743433) of different sizes (133, 236, 345 and $439 \mathrm{bp})$ was amplified using cassava cDNAs as a template and the corresponding primer pairs (Additional file 2: Table S3). Then the amplified fragments were cloned into pCsCMV-NC to generate pCsCMV$\mathrm{PDS}_{487}, \mathrm{pCsCMV}-\mathrm{ChlI}_{133},-\mathrm{ChlI}_{236},-\mathrm{ChlI}_{345}$ and $-\mathrm{ChlI}_{439}$ using Nimble Cloning [35]. In brief, 20-100 ng circular destination vector $(1-2 \mu \mathrm{L})$ and 10-50 ng PCR insert were added a PCR microtube containing $5 \mu \mathrm{L} 2 \times$ Nimble Mix for a final volume of $10 \mu \mathrm{L}$. The reaction mixture was incubated in a water bath for $1 \mathrm{~h}$ at $50{ }^{\circ} \mathrm{C}$ and then performed transformation in Escherichia coli strain DH5 $\alpha$. The accuracy of all resulting constructs was identified by sequencing.

\section{Plant growth and agroinfiltration}

Cassava plants were propagated vegetatively by planting properly lignified stem cuttings in soil. Cassava and Nicotiana benthamiana plants were grown in a greenhouse at $25{ }^{\circ} \mathrm{C}$ under a $16 / 8$-h photoperiod. 3-week and 5-monthold cassava plants, and 2-week-old N.benthamiana seeding were used for agroinoculation. For agroinfiltration of recombinant CsCMV clones, the CsCMV-NC-based constructs were transformed into Agrobacterium tumefaciens GV3101 with pSoup helper plasmid, respectively. A single colony of A. tumefaciens strain GV3101 for each viral construct were gown overnight in Luria-Bertani medium containing rifampicin $(25 \mathrm{mg} / \mathrm{L})$ and kanamycin $(50 \mathrm{mg} / \mathrm{L})$ at $28^{\circ} \mathrm{C}$. Subsequently, overnight bacterial cultures were centrifuged at $2,500 \mathrm{~g}$ for $10 \mathrm{~min}$ and were resuspended in agroinfiltration buffer $(10 \mathrm{mM} \mathrm{MgCl}$, $10 \mathrm{mM}$ 2-(N-Morpholino) ethanesulfonic acid [pH 5.5], and $100 \mu \mathrm{M}$ acetosyringone) for reaching an optical density of 0.8 at $600 \mathrm{~nm}\left(\mathrm{OD}_{600}\right)$ [42]. The agrobacterium suspension was kept at room temperature for $3 \mathrm{~h}$ in the dark before agroinfiltration. For cassava, the back sides of four healthy and fully developed leave in the middle of each plant were selected for agroinfiltration using a $1-\mathrm{mL}$ needleless syringe. Injections were performed at 8-10 spots on both sides of the main vein per leaf to enlarge the infiltrated leaf area. About $10 \mu \mathrm{L}$ of agrobacterium suspension was used for each spot. $N$. benthamiana leaves were agroinfiltrated as described previously [43].

\section{RT-PCR and RT-qPCR analysis}

Total RNA of cassava was extracted using the RNAprep Pure Plant Kit (Tiangen Biotech, China). For RT-PCR, the first-strand of cDNAs from $1.0 \mu \mathrm{g}$ of total RNA were synthesized with the Takara RNA PCR Kit (AMV) Ver. 3.0 (TaKaRa) using random 9 mers and oligo dT-Adaptor primers. To test the stability of the inserted target fragments in CsCMV-based vectors during viral infection, RT-PCR was performed using the primer pair CsCMV5416F(5'-TTGTAGCTGCCGTCCTAACTTGG$\left.3^{\prime}\right)$ /5730R (5'-ACCAAATTGGAGGCTGGCTTCA-3') flanking the NC frame. For RT-qPCR, three independent experiments were performed and each included six plants per treatment group. Two to three systemic leaves with symptomatic or silencing phenotypes in cassava plant agroinoculated with each CsCMV construct were pooled for total RNA extraction. The cDNAs from $1 \mu \mathrm{g}$ of DNAfree RNA and oligo (dT) using PrimeScript RT Reagent Kit (TaKaRa) were synthesized following the manufacturer's instructions. All RT-qPCR reactions were carried out using SYBR Premix EX Taq II Kit (TaKaRa). The cassava Mepp $2 A$ gene and $N$. benthamiana actin gene were used as an internal control for normalizing the expression of target genes. The species-specific primer pairs for PDS and ChlI (Additional file 2: Table S4) were used to test the silencing effect in each of these genes, and the expression level of each target gene was calculated using the deltadelta $\mathrm{Ct}$ method compared with the expression levels of the corresponding gene in the CsCMV-NC-infected samples [25]. The accumulation levels of CsCMV-CM and CsCMV-NC in systemically infected plants were quantified using the specific primers of CsCMV coat $(C P)$ gene (Additional file 2: Table S4).

\section{Statistical analyses}

Statistical analyses were performed using SPSS 23.0 (SPSS Inc., Chicago, IL, USA) software and all graphs were generated by Origin 2019 (OriginLab Corporation, Northampton, MA, USA). Statistical significance was assessed using Student's $t$ test, values of $P<0.05$ were considered statistically significant. Data were presented as the means \pm standard deviation (S.D.) of three biological replicates. 


\section{Supplementary Information}

The online version contains supplementary material available at https://doi. org/10.1186/s13007-021-00775-w.

\begin{abstract}
Additional file 1: Figure S1. Detection of viral RNA of the CSCMV-CM and CsCMV-NC in infected cassava leaves using RTPCR. Figure S2. Silencing phenotypes in 5-month-old cassava plants induced by infection with $\mathrm{pCsCMV-PDS_{487 }}$ or pCsCMV$\mathrm{ChIl}_{345}$ at 45 dpi. Figure S3. Alignment of the cassava 487bp PDS (GenBankaccession:XM_021757403) and 345-bp Chll gene fra gments(GenBankaccession:XM_021743433) in PCsCMV-PDS 487 and pCsCMV-ChIl ${ }_{345}$ with the N.benthamiana PDS (GenBankaccession: DQ469932) and Chll (SequencelD:Niben101Scf16898g00001.1) homologues by the Sol Genomics Network (SGN). (https://solgenomics.net/ organism/Nicotiana_benthamiana/genome). Figure S4. Detection of viral RNA of the CsCMV-CM and CsCMV-NC in cassava storage roots using RT-PCR.
\end{abstract}

Additional file 2: Table S1. Primers used in identification of the complete genome sequence of the CsCMV-CM. Table S2. Primers used in construction of agroinfectious clone PCsCMV-CM and PCsCMV-NC vector. Table S3. Primers used for Nimble Cloning of gene fragments into the PCsCMV-NC vector. Table S4. Primers used for quantitative RT-PCR analyses.

\section{Acknowledgements}

We thank Dr. Jie Cai and Dr. Weiwei Tie for providing cassava plant materials.

\section{Authors' contributions}

WS, DT and PZ conceived and designed the research plan; DT, PY, HC, YL and WL performed the experiments; $H W, X Y, D S$ and $X L$ analyzed the data; $W S$ and DT wrote the article. All authors read and approved the final manuscript.

\section{Funding}

This work was supported by the National Natural Science Foundation of China (grant no. 32000399) and Central Public Interest Scientific Institution Basal Research Fund for Chinese Academy of Tropical Agricultural Sciences (grant no.19CXTD-33,1630052019018 and 1630052019019).

\section{Declarations}

Ethics approval and consent to participate

Local, National and International guidelines were followed in this study with virus induced gene silencing in plants.

\section{Consent for publication}

Not applicable.

\section{Competing interests}

The authors declare that they have no competing interests.

\begin{abstract}
Author details
${ }^{1}$ Key Laboratory of Biology and Genetic Resources of Tropical Crops, Ministry of Agriculture and Rural Affairs \& Institute of Tropical Bioscience and Biotechnology, Chinese Academy of Tropical Agricultural Sciences, Haikou 571101, China. ${ }^{2}$ Hainan Key Laboratory for Protection and Utilization of Tropical Bioresources \&, Institute for Tropical Agricultural Resources, Chinese Academy of Tropical Agricultural Sciences, Haikou 571101, China. ${ }^{3}$ College of Plant Protection, Hainan University, Haikou 570228, China. ${ }^{4}$ College of Horticulture, Hainan University, Haikou 570228, China.
\end{abstract}

Received: 22 January 2021 Accepted: 1 July 2021

Published online: 12 July 2021

\section{References}

1. El-Sharkawy MA. Cassava biology and physiology. Plant Mol Biol. 2004:56:481-501.

2. Malik Al, Kongsil P, Nguyễn VA, Ou W, Sholihin, Srean P, et al. Cassava breeding and agronomy in Asia: 50 years of history and future directions. Breed Sci. 2020. 70:145-66.

3. Bredeson JV, Lyons JB, Prochnik SE, Wu GA, Ha CM, Edsinger-Gonzales $E$, et al. Sequencing wild and cultivated cassava and related species reveals extensive interspecific hybridization and genetic diversity. Nat Biotechnol. 2016;34:562-70.

4. Wang W, Feng B, Xiao J, Xia Z, Zhou X, Li P, et al. Cassava genome from a wild ancestor to cultivated varieties. Nat Commun. 2014;5:5110.

5. Anjanappa RB, Mehta D, Okoniewski MJ, Szabelska-Berȩsewicz A, Gruissem W, Vanderschuren $\mathrm{H}$. Molecular insights into cassava brown streak virus susceptibility and resistance by profiling of the early host response. Mol Plant Pathol. 2018;19:476-89.

6. Liao W, Yang Y, Li Y, Wang G, Peng M. Genome-wide identification of cassava R2R3 MYB family genes related to abscission zone separation after environmental-stress-induced abscission. Sci Rep. 2016;6:32006.

7. Ruan MB, Guo X, Wang B, Yang YL, Li W, Yu X, et al. Genome-wide characterization and expression analysis enables identification of abiotic stress-responsive MYB transcription factors in cassava (Manihot esculenta). J Exp Bot. 2017;68:3657-72.

8. Yan Y, Wang L, Ding Z, Tie W, Ding X, Zeng C, et al. Genome-wide identification and expression analysis of the mitogen-activated protein kinase gene family in cassava. Front Plant Sci. 2016;7:1294.

9. Li H-Q, Sautter C, Potrykus I, Puonti-Kaerlas J. Genetic transformation of cassava (Manihot esculenta Crantz). Nat Biotechnol. 1996;14:736-40.

10. Liu J, Zheng Q, Ma Q, Gadidasu KK, Zhang P. Cassava genetic transformation and its application in breeding. J Integr Plant Biol. 2011:53:552-69.

11. Gomez MA, Lin ZD, Moll T, Chauhan RD, Hayden L, Renninger K, et al. Simultaneous CRISPR/Cas9-mediated editing of cassava elF4E isoforms nCBP-1 and nCBP-2 reduces cassava brown streak disease symptom severity and incidence. Plant Biotechnol J. 2019;17:421-34.

12. Jørgensen K, Bak S, Busk PK, Sørensen C, Olsen CE, PuontiKaerlas J, et al Cassava plants with a depleted cyanogenic glucoside content in leaves and tubers distribution of cyanogenic glucosides, their site of synthesis and transport, and blockage of the biosynthesis by RNA interference technology. Plant Physiol. 2005;139:363-74.

13. Odipio J, Alicai T, Ingelbrecht I, Nusinow DA, Bart R, Taylor NJ. Efficient CRISPR/Cas9 genome editing of phytoene desaturase in cassava. Front Plant Sci. 2017;8:1780.

14. Ogwok E, Odipio J, Halsey M, Gaitán-Solís E, Bua A, Taylor NJ, et al. Transgenic RNA interference (RNAi)-derived field resistance to cassava brown streak disease. Mol Plant Pathol. 2012;13:1019-31.

15. Lange $M$, Yellina AL, Orashakova S, Becker A. Virus-induced gene silencing (VIGS) in plants: an overview of target species and the virusderived vector systems. Methods Mol Biol. 2013;975:1-14.

16. Dommes AB, Gross T, Herbert DB, Kivivirta KI, Becker A. Virus-induced gene silencing: empowering genetics in non-model organisms. J Exp Bot. 2019;70:757-70.

17. Beyene $G$, Chauhan RD, Taylor NJ. A rapid virus-induced gene silencing (VIGS) method for assessing resistance and susceptibility to cassava mosaic disease. Virol J. 2017;14:47.

18. Fofana IB, Sangare A, Collier R, Taylor C, Fauquet CM. A geminivirusinduced gene silencing system for gene function validation in cassava. Plant Mol Biol. 2004;56:613-24.

19. Lentz EM, Kuon JE, Alder A, Mangel N, Zainuddin IM, McCallum EJ, et al. Cassava geminivirus agroclones for virus-induced gene silencing in cassava leaves and roots. Plant Methods. 2018;14:73.

20. Zaidi SS, Vasudevan K, Lentz EM, Vanderschuren H. Virus-induced gene silencing (VIGS) in cassava using geminivirus agroclones. In: VirusInduced Gene Silencing in Plants: Methods Mol Biol. 2020; 2172: 51-64.

21. Brewer HC, Hird DL, Bailey AM, Seal SE, Foster GD. A guide to the contained use of plant virus infectious clones. Plant Biotechnol J. 2018;16:832-43.

22. Liu Y, Schiff M, Dinesh-Kumar SP. Virus-induced gene silencing in tomato. Plant J. 2002;31:777-86. 
23. Zeng HQ, Xie YW, Liu GY, Wei YX, Hu W, Shi HT. Agrobacterium-mediated gene transient overexpression and Tobacco rattle virus (TRV)based gene silencing in Cassava. Int J Mol Sci. 2019;20:3976.

24. Calvert LA, Cuervo MI, Ospina MD, Fauquet CM, Ramirez B-C. Characterization of cassava common mosaic virus and a defective RNA species. J Gen Virol. 1996;77:525-30.

25. Tuo DC, Zhao GY, Yan P, Li RM, Chen X, Wang WQ, et al. First report of cassava common mosaic virus infecting cassava in Mainland China. Plant Dis. 2020;104:997.

26. Zanini AA, Cuellar WJ, Celli MG, Luque AV, Medina RD, Conci VC, et al. Distinct strains of the re-emergent cassava common mosaic virus (genus: Potexvirus) infecting cassava in Argentina. Plant Pathol. 2018;67:1814-20.

27. Calvert LA, Thresh JM. The viruses and virus diseases of cassava. In. Edited by Hillocks RJ, Thresh JM, vol., edn. Wallingford: CABl; 2001: 237-60.

28. Bouton C, King RC, Chen H, Azhakanandam K, Bieri S, Hammond-Kosack $\mathrm{KE}$, et al. Foxtail mosaic virus: a viral vector for protein expression in cereals. Plant Physiol. 2018;177:1352-67.

29. Chapman $S$, Kavanagh T, Baulcombe D. Potato virus $X$ as a vector for gene expression in plants. Plant J. 1992;2:549-57.

30. Mellado-Sánchez M, McDiarmid F, Cardoso V, Kanyuka K, MacGregor DR. Virus-mediated transient expression techniques enable gene function studies in black-grass. Plant Physiol. 2020;183:455-9.

31. Sempere RN, Gomez P, Truniger V, Aranda MA. Development of expression vectors based on pepino mosaic virus. Plant Methods. 2011;7:6

32. Faivre-Rampant O, Gilroy EM, Hrubikova K, Hein I, Millam S, Loake GJ, et al. Potato virus $X$-induced gene silencing in leaves and tubers of potato. Plant Physiol. 2004;134:1308-16

33. Liu N, Xie K, Jia Q, Zhao J, Chen T, Li H, et al. Foxtail mosaic virus-induced gene silencing in monocot plants. Plant Physiol. 2016;171:1801-7.

34. Mei Y, Zhang C, Kernodle BM, Hill JH, Whitham SA. A foxtail mosaic virus vector for virus-induced gene silencing in maize. Plant Physiol. 2016;171:760-72
35. Yan P, Zeng Y, Shen W, Tuo D, Li X, Zhou P. Nimble cloning: a simple, versatile, and efficient system for standardized molecular cloning. Front Bioeng Biotechnol. 2020;7:460.

36. Hellens R, Mullineaux P, Klee $H$. Technical focus: a guide to agrobacterium binary Ti vectors. Trends Plant Sci. 2000;5:446-51.

37. Dickmeis C, Fischer R, Commandeur U. Potato virus X-based expression vectors are stabilized for long-term production of proteins and larger inserts. Biotechnol J. 2014;9:1369-79.

38. Dong Y, Burch-Smith TM, Liu Y, Mamillapalli P, Dinesh-Kumar SP. A ligationindependent cloning tobacco rattle virus vector for high-throughput virus-induced gene silencing identifies roles for NbMADS4-1 and -2 in floral development. Plant Physiol. 2007;145:1161-70.

39. Fernandez-Pozo N, Rosli HG, Martin GB, Mueller LA. The SGN VIGS tool: user-friendly software to design virus-induced gene silencing (VIGS) constructs for functional genomics. Mol Plant. 2015;8:486-8.

40. Safari F, Rahmani Barouji S, Tamaddon AM. Strategies for improving siRNA-induced gene silencing efficiency. Adv Pharm Bull. 2017;7:603-9.

41. Willemsen A, Zwart MP. On the stability of sequences inserted into viral genomes. Virus Evol. 2019;5:vez045.

42. Tuo DC, Fu LL, Shen WT, Li XY, Zhou P, Yan P. Generation of stable infectious clones of plant viruses by using Rhizobium radiobacter for both cloning and inoculation. Virology. 2017;510:99-103.

43. Du J, Rietman H, Vleeshouwers VGAA. Agroinfiltration and PVX agroinfection in potato and Nicotiana benthamiana. Jove-J Vis Exp. 2014:e50971.

\section{Publisher's Note}

Springer Nature remains neutral with regard to jurisdictional claims in published maps and institutional affiliations.
Ready to submit your research? Choose BMC and benefit from:

- fast, convenient online submission

- thorough peer review by experienced researchers in your field

- rapid publication on acceptance

- support for research data, including large and complex data types

- gold Open Access which fosters wider collaboration and increased citations

- maximum visibility for your research: over $100 \mathrm{M}$ website views per year

At BMC, research is always in progress.

Learn more biomedcentral.com/submissions 